\title{
Stability Problems for the Euler Equation on the 2-Dimensional Torus
}

\section{Piero Negrini}

Dipartimento Matematico "G. Castelnuovo", Sapienza, Università di Roma.

\begin{abstract}
We discuss the classical problem of the stability of the stationary solution $\psi^{*}=-\cos y$ for the Euler equation on a 2-dimensional flat torus of sides $2 \pi L$ and $2 \pi$. We prove that $\psi^{*}$ is linearly stable if $L \in(0,1)$ and that exponentially unstable modes occur in a right neighborhood of $L=n$ for any integer $n$.
\end{abstract}

This talk is based on the results of a joint work with my colleague Paolo Buttà $^{1}$. We consider a 2-dimensional torus $T^{2}(L)$ :

$$
T^{2}(L)=\left\{\left(z=(x, y) \in \mathbb{R}^{2}, \bmod (2 \pi L, 2 \pi)\right\}\right.
$$

where $L \in(0, \infty)$. On $T^{2}(L)$ we consider the Euler Equation written in terms of the stream function $\phi \in H^{2}\left(T^{2}(L), \mathbb{R}\right)$ :

$$
\frac{\partial \Delta \phi}{\partial t}+\langle J \nabla \phi, \nabla \Delta \phi\rangle=0
$$

(where $\langle J \nabla \phi, \nabla \Delta \phi\rangle=\frac{\partial \phi}{\partial y} \frac{\partial \Delta \phi}{\partial x}-\frac{\partial \phi}{\partial x} \frac{\partial \Delta \phi}{\partial y}$ ). As it is well known, equation (0.2) admits two independent first integrals:

$$
\begin{gathered}
I_{1}=\int_{T^{2}(L)} d x d y|\nabla \phi|^{2} \\
I_{2}=\int_{T^{2}(L)} d x d y(\Delta \phi)^{2}
\end{gathered}
$$

Any regular function defined on $T^{2}(L)$, depending either on the $y$ variable or on the $x$ variable is a stationary solution of $(0.2)$. More generally, any function $\phi$ such that $\Delta \phi$ is functionally dependent on $\phi$ is a stationary solution. Obviously the space spanned by the eigenfunctions of the Laplacian with eigenvalue -1 is a space of stationary solutions.

\footnotetext{
${ }^{1}$ Dipartimento Matematico "G. Castelnuovo", Sapienza, Università di Roma.
} 
We are interested in the dependence on $L$ of the stability properties of the stationary solution $\phi^{*}=-\cos y$ (a rather old problem). Setting

$$
\phi=-\cos y+\Psi
$$

we rewrite $(0.2)$ :

$$
\frac{\partial \Delta \Psi}{\partial t}+\sin y \frac{\partial}{\partial x}(\Psi+\Delta \Psi)+\langle J \nabla \Psi, \nabla \Delta \Psi\rangle=0
$$

The corresponding linear equation is:

$$
\frac{\partial \Delta \Psi}{\partial t}+\sin y \frac{\partial}{\partial x}(\Psi+\Delta \Psi)=0
$$

According to Arnol'd, the stationary solution $\Psi^{*}=0$ is unstable [resp. stable] for $L>1$ [resp. $0<L \leq 1$ ], and the proof of instability can be obtained using the same techniques already employed in the paper by Meshalkin and Sinai [10]. Previous announcement of this result was given in the report "A.N. Kolmogoroff's Seminar on selected problems of analysis" 2 by Arnol'd and Meshalkin [3]. We recall that these authors considered the (forced) Navier-Stokes equation,

$$
\frac{\partial \Delta \psi}{\partial t}+\langle J \nabla \psi, \nabla \Delta \psi\rangle=\nu \Delta^{2} \psi+\gamma \cos y
$$

and the stationary solution $\psi^{*}=-\frac{\gamma}{\nu} \cos y$. For the corresponding linearized equation,

$$
\frac{\partial \Delta \Psi}{\partial t}+\frac{\gamma}{\nu} \sin y \frac{\partial}{\partial x}(\Psi+\Delta \Psi)=\nu \Delta^{2} \Psi
$$

the following theorem was proved using techniques of continued fractions:

Theorem 0.1. Consider the stationary solution $\psi^{*}=-\frac{\gamma}{\nu} \cos y$. If $L<1$ then $\psi^{*}$ is stable for all $\gamma$, while for $L>1$ (and $\frac{\gamma}{\nu}$ fixed) one can always find $\nu_{0}>0$ such that the zero solution is exponentially unstable for $\nu<\nu_{0}$.

Note that the case $L=1$ is not included in the theorem.

Few years after the Meshalkhin-Sinai paper, V.I.Yudovich [11] employed the same techniques to study the bifurcation phenomenon occurring when there is a loss of stability of the laminar flow of a viscous incompressible fluid. In recent years Friedlander et al. [6], following the Arnol'd suggestion, studied the instability of the stationary solution $\phi^{(m)}=-\frac{1}{m} \cos m y, m \in \mathbb{N}$, to equation (0.2). The instability property relies on the existence of roots with positive real part of an infinite algebraic system of complex variable, with coefficients depending on $L$. These roots determine the exponential growth of the corresponding unstable modes of the linearized equation. In

\footnotetext{
$2^{2}$ The seminar topics can be read in the book: "Kolmogorov In Perspective", History of Mathematics, Vol 20, Am. Math. Soc.-London Math. Soc., (2000), pp 89-90.
} 
particular, for $m=1$, they proved the existence of real roots for $L>$ 1. Moreover, numerical calculations indicate that for any $L>1$ there is exactly one real root.

In this talk, after a preliminary discussion of the stability for $L \leq 1$, we present a new proof of the exponential instability of $\psi^{*}$ when $L>1$ (see Theorem 0.5 ). More precisely we analyze the existence of $2 \pi$-periodic solution of second order linear differential equation (0.27) (the well-known Rayleigh equation) whose coefficients depend on two parameters $(\omega, \varepsilon) \in$ $\mathbb{C} \times \mathbb{R}$. To this purpose we consider the functional system (0.30) which is solved by a perturbative approach, obtaining a unique solution $\omega=\omega(\varepsilon)$ defined in a right neighborhood of $\varepsilon=0$. This function ensures the existence of periodic solutions for the corresponding one parameter differential equation. Moreover, $\Re \omega(\varepsilon)$ gives the strenght of the exponential growth of the unstable mode of the linearized equation (0.6). We notice here that $\Re \omega(\varepsilon)=O(\varepsilon), \Im \omega(\varepsilon)=o(\varepsilon)$. However we are not able to prove the identity $\Im \omega(\varepsilon) \equiv 0$. The numerical result in [6] strongly support this conclusion, but till now the analytical proof is missing. ${ }^{3}$

We begin now to discuss the case $L \leq 1$. We observe that both equations (0.5), (0.6) admit the first integral

$$
\mathcal{I}:=\int_{T^{2}(L)} d x d y\left((\Delta \Psi)^{2}-|\nabla \Psi|^{2}\right)
$$

Let us emphasize that $\mathcal{I}$ is a positive, but not a positive-definite functional. Indeed, $\mathcal{I}$ is zero for $L<1$ on the space spanned by the functions $\sin y$, $\cos y$, while it is zero for $L=1$ also on the space spanned by the functions $\sin x, \cos x$. Therefore for $L \leq 1$ the first integral $\mathcal{I}$ is not sufficient in order to establish the stability of the null stationary solution neither for the nonlinear equation nor for the linear one.

From now on we concentrate on the latter and we remark that it admits also the family of first integrals $\mathcal{I}_{k_{2}}$ :

$$
\mathcal{I}_{k_{2}}:=\int_{T_{L}^{2}} d x d y e^{i k_{2} y} \Delta \Psi, \quad k_{2} \in \mathbb{Z}
$$

Writing $\Psi$ in Fourier expansion,

$$
\Psi(x, y)=\sum_{k \in Z^{2}} \Psi_{k_{1}, k_{2}} e^{i\left(\frac{k_{1}}{L} x+k_{2} y\right)},
$$

\footnotetext{
${ }^{3}$ In [10] it was proven that the analogous equation has only real roots.
} 
equation (0.6) becomes the following infinite system of O.D.E.,

$$
\begin{aligned}
{\left[\left(\frac{k_{1}}{L}\right)^{2}+k_{2}^{2}\right] \dot{\Psi}_{k_{1}, k_{2}}=} & \frac{k_{1}}{2 L}\left\{\left[\left(\frac{k_{1}}{L}\right)^{2}+k_{2}^{2}+2 k_{2}\right] \Psi_{k_{1}, k_{2}+1}\right. \\
& \left.-\left[\left(\frac{k_{1}}{L}\right)^{2}+k_{2}^{2}-2 k_{2}\right] \Psi_{k_{1}, k_{2}-1}\right\} .
\end{aligned}
$$

To each fixed $k_{1} \in \mathbb{Z}$ there corresponds a subsystem $\mathcal{S}_{k_{1}}$. We can represent $\mathcal{I}$ and $\mathcal{I}_{k_{2}}$ in terms of the Fourier coefficients,

$$
\begin{gathered}
\mathcal{I}=\sum_{\left(k_{1}, k_{2}\right) \in \mathbb{Z}^{2}}\left[\left(\frac{k_{1}}{L}\right)^{2}+k_{2}^{2}\right]\left[\left(\frac{k_{1}}{L}\right)^{2}+k_{2}^{2}-1\right]\left|\Psi_{k_{1}, k_{2}}\right|^{2}, \\
\mathcal{I}_{k_{2}}=-k_{2}^{2} \Psi_{0, k_{2}}, \quad k_{2} \in \mathbb{Z} .
\end{gathered}
$$

We notice that the first integral (0.12) is given by the sum $\sum_{k_{1} \in Z} \mathcal{I}_{k_{1}}$

$$
\mathcal{I}_{k_{1}}:=\sum_{k_{2} \in \mathbb{Z}}\left[\left(\frac{k_{1}}{L}\right)^{2}+k_{2}^{2}\right]\left[\left(\frac{k_{1}}{L}\right)^{2}+k_{2}^{2}-1\right]\left|\Psi_{\left(k_{1}, k_{2}\right)}\right|^{2}, k_{1} \in \mathbb{Z},
$$

each one of them being a first integral of the subsystem $\mathcal{S}_{k_{1}}$ we obtain from (0.11) by fixing $k_{1}$.

For each one of the subsystems $\mathcal{S}_{k_{1}}$ we have the two (possibly formal) first integrals $\mathcal{H}_{k_{1}}^{ \pm}$:

$$
\begin{array}{r}
\mathcal{H}_{k_{1}}^{+}:=\sum_{k_{2} \in Z}\left[\left(\frac{k_{1}}{L}\right)^{2}+k_{2}^{2}\right] \Psi_{k_{1}, k_{2}} \\
\mathcal{H}_{k_{1}}^{-}:=\sum_{k_{2} \in Z}(-1)^{k_{2}}\left[\left(\frac{k_{1}}{L}\right)^{2}+k_{2}^{2}\right] \Psi_{k_{1}, k_{2}}
\end{array}
$$

Then we have the following Theorem:

Theorem 0.2. If $0<L<1$ the zero solution of (0.6) is stable.

Proof. Assume $0<L<1$. In this case, see (0.12), the first integral $\mathcal{I}$ implies that all the Fourier coefficients $\Psi_{k_{1}, k_{2}}$ are controlled except the coefficients $\Psi_{0, \pm 1}$, which however are first integrals, see (0.13).

Remark 0.3. Of course, this result does not imply the stability of the zero solution for the nonlinear equation (0.5), because $\mathcal{I}_{k_{2}}$ is not a first integral with respect to this evolution. 
For $L=1$ even the problem of linear stability still is open. Indeed, again by virtue of (0.12) and (0.13), all the Fourier coefficients $\Psi_{k_{1}, k_{2}}$ are controlled, except $\Psi_{1,0}$. We remark that system $\mathcal{S}_{1}$ decouples now into the equation

$$
\dot{\Psi}_{1,0}=\frac{1}{2}\left[a_{1}-a_{-1}\right]
$$

and the two systems $\mathcal{S}_{1}^{+}$and $\mathcal{S}_{1}^{-}$defined respectively by the equations ${ }^{4}$ :

$$
\omega_{k_{2}} \dot{a}_{k_{2}}=a_{k_{2}+1}-a_{k_{2}-1} \text {, }
$$

where $\omega_{k_{2}}=\frac{k_{2}^{2}+1}{k_{2}^{2}}, a_{k_{2}}=k_{2}^{2} \Psi_{1, k_{2}}, a_{0}=0$ and $k_{2} \geq 1$ for $\mathcal{S}_{1}^{+}, k_{2} \leq-1$ for $\mathcal{S}_{1}^{-}$. Moreover $\mathcal{S}_{1}^{ \pm}$admits the first integral $\mathcal{I}_{1}^{ \pm}$:

$$
\mathcal{I}_{1}^{ \pm}:=\sum_{ \pm k_{2} \in \mathbb{N}} \omega_{k_{2}}\left|a_{k_{2}}\right|^{2}
$$

We could try to use $\mathcal{H}_{1}^{ \pm 5}$, see $(0.15)$, to obtain:

$$
\left|\Psi_{1,0}\right| \leq \sum_{k_{2} \neq 0} \omega_{k_{2}}\left|a_{k_{2}}\right|+\left|\mathcal{H}_{1}^{+}\right|+\left|\mathcal{H}_{1}^{-}\right|
$$

However $\mathcal{I}_{1}^{ \pm}$do not make sure the convergence of the series in the r.h.s. of $(0.19)$ as the space $\ell_{1}$ is not a subset of the space $\ell_{2}$.

Obviously the first integrals $\mathcal{I}_{1}^{ \pm}$prevent the exponential instability of the zero solution of system $\mathcal{S}_{1}$. However, the secular instability could occur. Recalling the strong relations between Euler equations and the Euler-Poisson equations for the rigid body (see [4], [8]), in our opinion the case $L=1$ could be the analogous of the symmetric case:

$$
\begin{aligned}
& \dot{\Omega}_{1}=(C-A) \Omega_{2} \Omega_{3} \\
& \dot{\Omega}_{2}=(A-C) \Omega_{1} \Omega_{3} \\
& \dot{\Omega}_{3}=0
\end{aligned}
$$

Any stationary solutions $(p, q, 0)$ is unstable (not exponentially unstable) and the solutions of the corresponding linear equations have a secular growth.

\footnotetext{
${ }^{4}$ Systems of infinite linear equations related in some sense to the Euler Equation were studied in [9]. However (0.17) seems to belong to a different class of difficulty.

${ }^{5}$ In fact system $\mathcal{S}_{1}^{ \pm}$admits also the (possibly formal) first integral $\mathcal{H}_{o}^{ \pm}$:

$$
\mathcal{H}_{o}^{ \pm}:=\sum_{ \pm k_{2} \in \mathbb{N}_{0}} \omega_{2 k_{2}+1} a_{2 k_{2}+1}
$$
}


We are not able to prove the existence of solutions to $\mathcal{S}_{1}^{ \pm}$in the space $\ell_{2}$ such that

$$
\lim \sup _{t \rightarrow \infty}\left|\int_{0}^{t} d \tau\left[a_{1}(\tau)-a_{-1}(\tau)\right]\right|=\infty
$$

Of course, we can consider the following solution:

$$
a_{k_{2}}= \begin{cases}0, & k_{2} \leq-1 \\ 0, & k_{2}=2 n, n \geq 1 \\ 1, & k_{2}=2 n+1, n \geq 0\end{cases}
$$

so that $\Psi_{1,0}=t$. Then $\Psi=e^{i x}\left[t+\sum_{n \geq 0} \frac{1}{(2 n+1)^{2}} e^{i(2 n+1) y}\right]$ does not belong to $H^{2}\left(T^{2}(L), \mathbb{R}\right)$, and $\mathcal{I}$ becomes meaningless.

Remark 0.4. Let $a_{k_{2}}=0, \forall k_{2} \leq 0$. We define the sequence of functions $\left\{A_{k_{2}}\right\}_{k_{2} \geq 1}$ :

$$
A_{k_{2}}(t)=\int_{0}^{t} d \tau a_{k_{2}}(\tau)
$$

and we consider the integral system

$$
\omega_{k_{2}}\left[a_{k_{2}}(t)-a_{k_{2}}(0)\right]=A_{k_{2}+1}-A_{k_{2}-1},
$$

obtained from (0.17) for $k_{2} \geq 1$. Recall that the sequence of functions $\left\{\left|a_{k_{2}}(t)\right|\right\}$ is uniformly bounded, see (0.18). Therefore necessarly $A_{2}(t)$ is bounded and therefore also $A_{2 k_{2}}(t)$, is bounded for any natural $k_{2}$, while $A_{2 k_{2}+1}(t)$ is bounded for $k_{2} \geq 1$ if and only if $A_{1}(t)$ is bounded.

From (0.22) we easily get:

$$
\sum_{k_{2} \in \mathbb{N}} \omega_{2 k_{2}}\left[a_{2 k_{2}}(t)-a_{2 k_{2}}(0)\right]=-A_{1}(t)
$$

Therefore we can conclude that the divergence of the series in the l.h.s. is a necessary condition for the secular instability ${ }^{6}$.

We consider now the case $L>1$. We have the following instability theorem:

Theorem 0.5. The zero solution of equation (0.6) is exponentially unstable for $L$ belonging to a right neighborhood of $L=1$. Moreover, there exists a number $L_{0}, L_{0} \geq 1$, such that the zero solution is unstable for all $L>L_{0}$.

Theorem 0.5 is a straightforward consequence of the following main theorem.

\footnotetext{
${ }^{6}$ Recall however that $\left|a_{2 k_{2}}(t)-a_{2 k_{2}}(0)\right|$ is bounded for any $k_{2} \in \mathbb{N}$
} 
Theorem 0.6. The set $L=k, k \in \mathbb{N}$, is a bifurcation set. More precisely, there exist $\varepsilon_{0}>0$, a function $\omega^{+} \in C^{1}\left(\left[\left(0, \varepsilon_{0}\right) ; \mathbb{C}\right)\right.$ with $\Re\left(\omega^{+}(\varepsilon)\right) \in[\varepsilon, 3 \varepsilon]$ for all $\varepsilon \in\left(0, \varepsilon_{0}\right)$, and a solution to (0.6) of the form:

$$
\Psi^{+}(x, y, t)=e^{\frac{k}{L_{k}(\varepsilon)}\left(i x+\omega^{+}(\varepsilon) t\right)} f^{+}(y, \varepsilon),
$$

Moreover there exists a function $\omega^{-} \in C^{1}\left(\left[\left(0, \varepsilon_{0}\right) ; \mathbb{C}\right)\right)$ with $-\Re\left(\omega^{-}(\varepsilon)\right) \in$ $[\varepsilon, 3 \varepsilon]$ for all $\varepsilon \in\left(0, \varepsilon_{0}\right)$, and a solution of the form

$$
\Psi^{-}(x, y, t)=e^{\frac{k}{L_{k}(\varepsilon)}\left(i x+\omega^{-}(\varepsilon) t\right)} f^{-}(y, \varepsilon) .
$$

Finally functions $f^{ \pm}(\cdot, \varepsilon) \in C^{2}\left(S^{1} ; \mathbb{C}\right)$ are independent of $k$ and $L_{k}(\varepsilon):=$ $k(1+\varepsilon), k \in \mathbb{N}$.

Remark 0.7. We emphasize that the above result also implies that the number of unstable modes for the zero solution is diverging as $L \rightarrow \infty$. Note in fact that, letting $n=n(L)$ be the largest positive integer such that $\frac{L}{[L]-n}<1+\varepsilon_{0}$, we have $n(L) \rightarrow \infty$ as $L \rightarrow \infty$, and for each $k=$ $[L]-n, \ldots,[L]$ there exists $\varepsilon_{k} \in\left(0, \varepsilon_{0}\right)$ such that $k\left(1+\varepsilon_{k}\right)=L$. There are therefore $n+1$ unstable modes. This growth of unstable modes is the analogous to that one for the Navier-Stokes Equation. Indeed the dimension of the unstable manifold of the Kolmogorov flow is equal to $2[L]$ (see [5]).

Let us sketch the main steps of the proof of Theorem (0.6), the detailed proof can be found in [7]. Given $\varepsilon \in(0,1)$ and $\omega \in \mathbb{C}$, a function of the form

$$
\Psi(x, y, t)=e^{\frac{1}{1+\varepsilon}(i x+\omega t)} f(y)
$$

is a solution to ( 0.6$)$ if and only if $f(y)$ is a $2 \pi$-periodic solution of the Rayleigh equation

$$
\frac{d^{2} f}{d y^{2}}-\frac{i \omega+\varepsilon(2+\varepsilon) \sin y}{(1+\varepsilon)^{2}(i \omega-\sin y)} f=0 .
$$

Our strategy is to prove by a perturbative method that this solution does exist at least for small $\varepsilon$ and $\omega$. It is not restrictive to assume $f(0)=1$. Let $f(y, \omega, \beta, \varepsilon), \beta \in \mathbb{C}$, be the solution to $(0.27)$ with $f(0)=1$ and $f^{\prime}(0)=\beta$. Clearly $f(y, \omega, \beta, \varepsilon)$ solves the integral equation

$$
f(y)=1+\beta y+\int_{0}^{y} d \tau(y-\tau) G(\tau, \omega, \varepsilon) f(\tau) .
$$

where

$$
G(\tau, \omega, \varepsilon):=\frac{i \omega+\varepsilon(2+\varepsilon) \sin \tau}{2 \pi(1+\varepsilon)^{2}(i \omega-\sin \tau)} .
$$


By imposing periodic boundary condition we obtain the following system of equations in the variables $(\omega, \beta, \varepsilon)$,

$$
\left\{\begin{array}{l}
\int_{0}^{2 \pi} d \tau G(\tau, \omega, \varepsilon) f(\tau, \omega, \beta, \varepsilon)=0 \\
\int_{0}^{2 \pi} d \tau \tau G(\tau, \omega, \varepsilon) f(\tau, \omega, \beta, \varepsilon)=\beta,
\end{array}\right.
$$

Our purpose is to solve the system $(0.30)$ with respect to $(\omega, \beta)$, getting functions $\omega=\omega(\varepsilon), \beta=\beta(\varepsilon)$ (defined in a right neighborhood of $\varepsilon=0$ ). Setting

$$
R(y, \omega, \beta, \varepsilon):=f(y, \omega, \beta, \varepsilon)-1-\beta y,
$$

we rewrite $(0.30)$ as follows,

$$
\left\{\begin{array}{l}
\mathcal{E}_{0}(\omega, \varepsilon)+\mathcal{E}_{1}(\omega, \varepsilon) \beta+A_{0}(\omega, \beta, \varepsilon)=0 \\
\mathcal{E}_{1}(\omega, \varepsilon)+\mathcal{E}_{2}(\omega, \varepsilon) \beta+A_{1}(\omega, \beta, \varepsilon)=\beta
\end{array}\right.
$$

where

$$
\begin{array}{r}
\mathcal{E}_{n}(\omega, \varepsilon)=\int_{0}^{2 \pi} d \tau \tau^{n} G(\tau, \omega, \varepsilon), n=0,1,2, \\
A_{n}(\omega, \beta, \varepsilon)=\int_{0}^{2 \pi} d \tau \tau^{n} G(\tau, \omega, \varepsilon) R(\tau, \omega, \beta, \varepsilon), n=0,1 .
\end{array}
$$

We notice that $\mathcal{E}_{0}(i z, \varepsilon)$ is zero for $z \in(0,1)$. Due to this degeneracy the standard implicit function theorem does not apply. To overcome this difficulty we exploit a fixed point argument in a cone

$$
\mathcal{K}_{\frac{1}{2}}:=\left\{\omega \in \mathbb{C}: 0<\Re \omega<\frac{1}{2},|\Im \omega| \leq|\Re \omega|\right\} .
$$

Plugging the second equation of system (0.32) into the first we can rewrite the latter in the form

$$
\omega=T_{\beta, \varepsilon}(\omega),
$$

where

$$
\begin{aligned}
T_{\beta, \varepsilon}(\omega):= & \omega-\mathcal{E}_{0}(\omega, \varepsilon)-\mathcal{E}_{1}(\omega, \varepsilon)^{2}-\mathcal{E}_{1}(\omega, \varepsilon) \mathcal{E}_{2}(\omega, \varepsilon) \beta \\
& -\mathcal{E}_{1}(\omega, \varepsilon) A_{1}(\omega, \beta, \varepsilon)-A_{0}(\omega, \beta, \varepsilon) .
\end{aligned}
$$

We have the following Lemma:

Lemma 0.8. There exists $\varepsilon_{0}^{\prime}>0$ such that, for any $|\beta| \leq 1$ and $\varepsilon \in\left(0, \varepsilon_{0}^{\prime}\right)$, the compact set $S_{\varepsilon}:=\{\omega \in \mathcal{K}: \varepsilon \leq \Re \omega \leq 3 \varepsilon\}$ is invariant under the map $T_{\beta, \varepsilon}$ and $T_{\beta, \varepsilon}$ is a contraction on $\bar{S}_{\varepsilon}$. Therefore, the fixed point equation 
(0.33) can be solved for any $|\beta| \leq 1$ and $\varepsilon \in\left(0, \varepsilon_{0}^{\prime}\right)$, getting $\omega=\bar{\omega}(\beta, \varepsilon)$ with $\varepsilon \leq \Re \bar{\omega}(\beta, \varepsilon) \leq 3 \varepsilon$.

Plugging $\omega=\bar{\omega}(\beta, \varepsilon)$ into the second equation of system $(0.32)$, the latter assume the form

$$
\beta=Q_{\varepsilon}(\beta) .
$$

Lemma 0.9. There exists $\varepsilon_{0} \in\left(0, \varepsilon_{0}^{\prime}\right]$ such that, for any $\varepsilon \in\left(0, \varepsilon_{0}\right)$ the unit disk $\{\beta \in C:|\beta| \leq 1\}$ is invariant under the map $Q_{\varepsilon}$ and $Q_{\varepsilon}$ is a contraction on it. Therefore, the fixed point equation (0.35) can be solved for $\varepsilon \in\left(0, \varepsilon_{0}\right)$, getting $\beta=\beta(\varepsilon)$.

The two lemmata allow to achieve the proof of Theorem (0.6) defining $\omega^{+}(\varepsilon):=\bar{\omega}(\beta(\varepsilon), \varepsilon)$. Completely analogous is the proof of the existence of $\omega^{-}(\varepsilon)$.

Finally the claim of the theorem is proved.

Remark 0.10. One can easily verify that any $2 \pi$-periodic solution to the Rayleigh equation (0.27) must satisfies the following conditions:

$$
\left\{\begin{array}{l}
\int_{0}^{2 \pi} d y\left\{\left|\frac{d f(y)}{d y}\right|^{2}+\frac{|\omega|^{2}+\Im \omega \sin y[1-\varepsilon(\varepsilon+2)]-\varepsilon(\varepsilon+2) \sin ^{2} y}{(1+\varepsilon)^{2}\left[\Re \omega^{2}+(\Im \omega+\sin y)^{2}\right]}|f(y)|^{2}\right\} \\
\Re \omega \int_{0}^{2 \pi} d y \frac{\sin y}{\left[\Re \omega^{2}+(\Im \omega+\sin y)^{2}\right]}|f(y)|^{2}=0
\end{array}\right.
$$

where $|f(y)|^{2}=f(y) \bar{f}(y)$ and analogously for $\left|\frac{d f(y)}{d y}\right|^{2}$ and $|\omega|^{2}$. Assuming $\Re \omega \neq 0$ we get

$$
\left\{\begin{array}{l}
\int_{0}^{2 \pi} d y\left\{\left|\frac{d f(y)}{d y}\right|^{2}+\frac{|\omega|^{2}-\varepsilon(\varepsilon+2) \sin ^{2} y}{(1+\varepsilon)^{2}\left[\Re \omega^{2}+(\Im \omega+\sin y)^{2}\right]}|f(y)|^{2}\right\}=0 \\
\int_{0}^{2 \pi} d y \frac{\sin y}{\left[\Re \omega^{2}+(\Im \omega+\sin y)^{2}\right]}|f(y)|^{2}=0
\end{array}\right.
$$

If $\varepsilon \in(-1,0]$ the first equality holds only for $f \equiv 0$. Then, we arrive again at the same conclusion already obtained discussing the case $L \leq 1$.

\section{References}

[1] Arnol'd, V., Mathematical Methods of Classical Mechanics, Springer, 1977.

[2] Arnol'd, V., Topological Methods in Hydrodynamics, Springer, 1998.

[3] Arnol'd, V. and Meshalkin, L.D., A.N. Kolmogoroff's Seminar on selected problems of analysis (1958-1959), Uspekhi Mat. Nauk, vol. XV, (91). (English Translation: Russian Mathematical Surveys)

[4] Arnol'd, V., Sur la géometrie différentielle des groupes de Lie de dimension infinie et ses applications à l'hydrodynamique des fluides parfaits, Ann. Inst. Fourier (Grenoble), 1966, vol. XXVI, pp. 319-361. 
[5] Babin, A.V. and Vishik, A.A., Attractors of Evolution Equations, North-Holland Publishing, 1992.

[6] Belenkaya, L., Friedlander, S. J. and Yudovich, V., The unstable spectrum of oscillating shear flows, Siam J. Appl. Math., 1999, vol. 59,pp. 1701-1715.

[7] Buttà, P. and Negrini, P., On the Stability Problem of Stationary Solutions for the Euler Equation on a 2-dimensional Torus, Mathematical Physics Preprint Archive, $m p_{-} a r c$, 2010, number 10-21. Available on line at: http : //www.ma.utexas.edu/mp_arc/index - 10.html.

[8] Fedorov, Yu.N. and Kozlov, V.V., Various aspect of $n$-dimensional rigid body dynamics in Dynamical Systems in Classical Mechanics, Advances in the Mathematical Sciences, AMS Translations, Series 2, 1995, vol. 168.

[9] Mattingly, J.C., Suidan, T. and Vanden-Eijden, E., Simple Systems with Anomalous Dissipation, Commun. Math. Phys., 2007, vol. 276, pp. 189-220

[10] Meshalkin, L.D. and Sinai, Ya.G., Investigation of the stability of a stationary solution of a system of equations for the plane movement of an incompressible viscous liquid, Prikl. Mat. Meh., 1961, vol. 25, pp. 1140-1143 (in Russian); translated as J. Appl. Math. Mech., 1961, vol. 25, pp. 1700-1705.

[11] Yudovich, V., Example of the generation of a secondary stationary or periodic flow when there is a loss of stability of the laminar flow of a viscous incompressible fluid., Prikl. Mat. Mex., 1965, 29, pp. 453-467 (in Russian); translated as J. Appl. Math.Mech., 1965, vol. 29, issue 3, pp.527-544. 